\title{
Administración electrónica en las universidades públicas españolas
}

\author{
Rocío Rocha*, Ángel Cobo**, Margarita Alonso*
}

Resumen: En este trabajo se analiza el nivel de penetración de la administración electrónica en el sistema universitario español a través de una evaluación heurística de las sedes electrónicas universitarias. Estas sedes se convierten en un elemento clave en los procesos de difusión de información y prestación de servicios electrónicos propios de una administración pública. Del trabajo se desprende una buena predisposición de las universidades ante la e-administración aunque su evolución aún no ha alcanzado unos niveles óptimos. Igualmente se analizan las principales características diferenciadoras de las instituciones de educación superior con respecto a otras administraciones y sus implicaciones desde el punto de vista de la administración electrónica.

Palabras clave: e-administración, sede electrónica, universidad, información, accesibilidad, usabilidad.

\section{The e-administration at public universities in Spain}

\begin{abstract}
The study analyses the level of penetration of electronic administration in the Spanish university system through a heuristic evaluation of university electronic sites. These sites are a key element in the process of disseminating information and the provision of services in public administration. The study highlights that universities have implemented e-administration to a significant degree although they have yet to reach optimal levels. The study also analyses the principal characteristics differentiating higher education institutions from other public administrations and what this implies for e-administration.
\end{abstract}

Keywords: e-administration, electronic headquarters, university, information, accessibility, usability.

\section{Introducción}

Las tecnologías de la información y las comunicaciones (TIC) se han instalado de forma vertiginosa en todos los ámbitos de nuestra sociedad, favoreciendo la evolución progresiva y creciente de la sociedad de la información. Las admi-

* Departamento de Administración de Empresas. Facultad de Ciencias Económicas y Empresariales. Universidad de Cantabria. Correo-e: rochar@unican.es; alonsom@unican.es.

** Departamento de Matemática Aplicada y Ciencias de la Computación. Facultad de Ciencias Económicas y Empresariales. Universidad de Cantabria. Correo-e: acobo@unican.es.

Recibido: 21-03-2011; 2. ${ }^{a}$ versión: 23-04-2011; aceptado: 09-05-2011. 
nistraciones públicas están llamadas a desempeñar un papel fundamental para fomentar el acercamiento de los ciudadanos a los servicios proporcionados por las TIC (Guillén, 2010), y las TIC deben contribuir a la mejora de la eficacia de las administraciones. En particular, las instituciones de educación superior deben desempeñar un doble papel, por un lado formar a profesionales que contribuyan a la potenciación de la sociedad de la información y por otro estar en la vanguardia del uso de la tecnología en su vínculo con los ciudadanos.

El presente trabajo trata de examinar la situación actual del sistema universitario público español respecto a la aplicación efectiva de las técnicas de e-administración y explorar la forma en que Internet es utilizada por las administraciones públicas universitarias. Para ello, se ha estructurado de la siguiente manera: tras esta primera introducción se comienza por estudiar el contexto en el que se inserta el estudio evaluativo realizado, así se presenta el marco regulatorio en España de la e-administración y se analizan las particularidades del sistema universitario español y los condicionantes actuales para la plena penetración de la e-administración. A continuación se indican los objetivos y metodología utilizada para la evaluación, para pasar a presentar los resultados del estudio realizado y sus conclusiones.

\section{La e-administración y su entorno regulatorio}

La administración electrónica comprende el uso de las nuevas tecnologías en las administraciones públicas, aunque, por supuesto, no es simplemente traspasar a medios electrónicos los procedimientos clásicos basados en papel, ni de utilizar las TIC como apoyo al trabajo. La administración tiene que integrarse plenamente en las TIC para ser una verdadera administración electrónica.

El marco regulatorio europeo de la e-administración abarca en la actualidad un amplio conjunto de normas que establecen pautas de actuación de obligado cumplimiento para las administraciones públicas de los estados miembros de la Unión Europea. Tomando como punto de partida la Directiva Europea 1999/93/ CE por la que se establecía un marco comunitario para la firma electrónica, se ha ido generando nueva normativa europea que ha tenido su reflejo en normativa de ámbito estatal, autonómico, local y municipal en España. A nivel nacional destaca especialmente la Ley 11/2007, de 22 de junio de 2007, de acceso electrónico de los ciudadanos a los servicios públicos (LAECSP), que obliga a las administraciones públicas a proporcionar el acceso a la información y servicios de su competencia, dotándose de los medios y sistemas electrónicos adecuados, y reconoce el derecho de los ciudadanos a relacionarse por medios electrónicos con las administraciones públicas. Este derecho ya aparecía en el artículo 35 de la Ley 30/1992 de Régimen Jurídico de las Administraciones Públicas y del Procedimiento Administrativo Común.

Con objeto de facilitar a los ciudadanos el acceso a los servicios de e-administración, la LAECSP introduce el concepto de sede electrónica como elemento 
clave e integrador de servicios. En su artículo 10.1 define una sede electrónica como "dirección electrónica disponible para los ciudadanos a través de redes de telecomunicaciones cuya titularidad, gestión y administración corresponde a una Administración Pública, órgano o entidad administrativa en el ejercicio de sus competencias". Más allá de una definición tan simple, una sede electrónica puede verse como una solución que sirve de puerta de acceso al ciudadano para toda aquella información y servicios on-line que se ponen a su disposición de forma actualizada y, preferentemente, personalizada. Conviene diferenciar claramente entre Web o portal institucional y sede electrónica; esta última debe caracterizarse por una serie de servicios y una serie de requerimientos formales que la propia LAECSP establece. Así por ejemplo, LAECSP establece la información mínima que debe aparecer en cualquier sede electrónica: identificación del titular de la sede, autoridades competentes, sistemas de firma electrónica admitidos, los distintos tipos de escritos, comunicaciones o solicitudes que pueden presentarse, los medios disponibles para la formulación de sugerencias y quejas, información sobre plazos y días hábiles. Otro aspecto importante en el que incide la legislación de la administración electrónica es el cumplimiento de criterios de accesibilidad y usabilidad.

A pesar de que la LAECSP obliga a las administraciones públicas a dotarse de los medios y sistemas electrónicos necesarios para que los ciudadanos puedan ejercer derechos reconocidos, trata de evitar invadir competencias de las Comunidades Autónomas y deja abierta su fecha límite de aplicación a las disponibilidades presupuestarias (RD 1671/2009 de 6 de noviembre). Esto ha llevado consigo, teniendo en cuenta la actual coyuntura económica, un desigual nivel de penetración de la e-administración en las administraciones públicas, y muy en particular en las instituciones de educación superior.

\section{La e-administración en el sistema universitario público español}

Las universidades españolas están inmersas en un profundo proceso de modernización para adaptarse a las nuevas demandas docentes, de investigación y transferencia de conocimiento, y de la integración en el Espacio Europeo de Educación Superior (EEES). El hecho de que las universidades hayan sido tradicionalmente instituciones punteras en innovación y aplicación de técnicas y tecnologías emergentes hace que estudiar la forma en la que éstas exponen su información en la Web y prestan servicios de administración electrónica pueda ser una interesante visión, no sólo de su forma de gestionar la información en la actualidad, sino un anticipo del futuro inmediato en otras administraciones o sectores menos innovadores.

Las universidades cuentan a priori con una serie de ventajas para la implementación efectiva de modelos de e-administración. Además del hecho ya comentado de ser instituciones punteras en innovación, el nivel de formación y uso 
de las TIC es superior a la media, tienen buenas dotaciones de medios informáticos, y su colectivo principal de administrados está claramente definido e identificado. Otra ventaja es la informatización de las principales áreas de negocio ya efectuada desde hace años, así por ejemplo, hoy en día la mayor parte de las universidades tienen procesos de matrícula on-line, consulta de expedientes, etc. Algunas de estas ventajas, pueden volverse en contra de las propias universidades, así por ejemplo, la existencia de sistemas de identificación de sus colectivos basados en firma sencilla (usuario/password), ha hecho menos urgente la aplicación de sistemas basados en certificados y la implantación de sistemas de firma electrónica avanzada. Además, según Bárcena, la introducción de la e-administración en las universidades es un proyecto costoso que precisa de grandes inversiones para modernizar y adaptar los sistemas de información y de importantes transformaciones organizativas (Bárcena, 2010).

Para que la e-administración se implante en una universidad, se recomienda el trabajo coordinado con otras instituciones. Es por ello que, la Intersectorial CRUE/TIC, promovida por la Conferencia de Rectores de las Universidades Españolas (CRUE), viene mostrando en los últimos años un gran interés en examinar la evolución del uso de las TIC en los ámbitos de actuación de las universidades, y muy especialmente en los aspectos relacionados con la administración electrónica. Esta Intersectorial CRUE/TIC realiza anualmente, desde 2006, un informe de la evolución de las TIC en el sistema universitario español, con el propósito de conocer la situación de las TIC en las universidades desde seis ejes diferentes, a efectos de este trabajo interesa especialmente el tercer eje estratégico, el correspondiente a gestión universitaria. En la última edición de este informe (UniversiTIC, 2010) participaron 71 universidades españolas, 49 de ellas públicas, representando en total el 93,7\% de la población de estudiantes universitarios españoles. A partir de los datos del informe se puede comprobar como las universidades continúan automatizando sus procesos de administración buscando con ello un incremento de la eficiencia con que se realizan este tipo de tareas. En particular, el porcentaje de procesos de administración que se encuentran automatizados es del 73\%, siendo los procesos más informatizados los referentes a gestión académica, de nóminas y tercer ciclo. Se observa también en el informe un notable esfuerzo en busca de la agilización y modernización de la atención a los usuarios con tecnologías propias de la administración electrónica, con una progresión positiva con respecto a años anteriores. El porcentaje medio de tecnologías propias de la administración electrónica en explotación en las universidades españolas es del 67\%. La pasarela de pago electrónico está presente en el 70\% de las universidades, y la firma digital es empleada ya por la mitad de universidades y un $37 \%$ de instituciones están en vías de utilizarla. Destaca igualmente que el 50\% de las universidades se han dotado de un marco regulador o normativa para la administración electrónica, aunque se debe mejorar en la implantación de sistemas de administración de la seguridad, ya que solo un 15\% lo tiene formalizado.

A nivel europeo una iniciativa destacada es el Proyecto Europeo STORK (Secure idenTity acrOss boRded linKed), iniciativa para facilitar la movilidad de es- 
tudiantes y facilitar a estudiantes extranjeros el acceso a servicios administrativos on-line, usando sus identidades electrónicas de origen. En este proyecto participan 29 miembros entre gobiernos nacionales, instituciones académicas y de investigación, y organizaciones privadas de Austria, Estonia, Italia, Portugal y España con el propósito principal de demostrar que la infraestructura puede ser usada para elaborar servicios con la garantía de mecanismos de autentificación internacional. Este proyecto está previsto que finalice a finales de mayo de 2011, y aunque está orientado inicialmente a estudiantes, sus resultados serán extensibles a servicios orientados a ciudadanos en general.

A pesar de todo lo expuesto, aún existen obstáculos que impiden una implantación efectiva de la e-administración; entre ellos, la falta de cultura organizativa, medios TIC, confianza en la seguridad, la complejidad, etc. Por otro lado, la implantación en las universidades depende mucho de las Comunidades Autónomas en las que están situadas, en el caso de comunidades grandes o "ricas" o con liderazgo en esta materia éstas "empujan" o apoyan a sus universidades; en cambio, en las pequeñas o con poco liderazgo, la evolución no está siendo tan efectiva, y la actual coyuntura económica ha venido a agravar la situación. Por otro lado, en el profundo cambio que se está produciendo en el sistema universitario español, las universidades han priorizado la adaptación al EEES con los consiguientes cambios es sus normativas y procesos de administración, dejando en ocasiones en un segundo plano los aspectos relativos a e-administración. En cualquier caso, todo el sistema universitario español trata, en definitiva, de aprovechar la oportunidad que la Ley $11 / 2007$ brinda a las universidades para revisar sus procesos administrativos, rediseñarlos de acuerdo con las nuevas tecnologías y planificar los proyectos para aplicar ese nuevo diseño.

\section{Objetivos y metodología del estudio de las sedes electrónicas universitarias}

Estudiar la forma en que las universidades presentan su información en la Web y el nivel de penetración de la administración electrónica en ellas resulta muy interesante, ya que ayuda a tener una visión, no sólo de su forma de administración, sino un anticipo del futuro inmediato en otros sectores menos innovadores (García, 2010). Por otro lado, puede ser relevante la detección de problemas recurrentes en el diseño de una sede electrónica universitaria que puedan perjudicar al ciudadano en su intento de acceso a la información.

En este trabajo se ha buscado comprobar si las universidades públicas españolas cuentan con sedes electrónicas para la difusión de información y la prestación de servicios electrónicos de una administración pública. Se ha tomado como referencia una serie de indicadores del marco normativo que regula el concepto de sede electrónica y se ha analizado igualmente el grado de accesibilidad y usabilidad de cada una de las sedes, para observar si la sede proporciona la facilidad necesaria para el acceso de manera legible, coherente, comprensible y funcional. 
Se analizaron un total de 50 portales webs de universidades públicas españolas, obteniendo como resultado, en el momento de realizar este trabajo (febrero, 2011), 17 portales con sede electrónica como tal, lo que supone un 34\% del total. De las 17 sedes electrónicas, 11 corresponden a universidades andaluzas y catalanas, comunidades que han respaldado notablemente el proceso de implantación de la e-administración. En cualquier caso, la mayoría de las universidades que aún no cuentan con sede electrónica han constituido grupos de trabajo para la elaboración de planes de implementación de la e-administración en sus organizaciones.

Una vez identificadas las 17 sedes electrónicas, para realizar el estudio se utilizó el análisis de expertos o evaluación heurística basada en la observación como metodología de trabajo (González y otros, 2001). La metodología toma como objeto empírico el acceso rápido y fácil a los distintos portales webs, no requiere la inversión de demasiados medios y favorece una cierta automatización de la recogida de datos (Aibar y otros, 2004). Cada una de las 17 sedes electrónicas fue objeto de una rigurosa evaluación de un total de 70 indicadores relativos a información y servicios proporcionados, accesibilidad y usabilidad.

La evaluación de las sedes corrió a cargo de tres expertos de la Universidad de Cantabria especializados en diseño y arquitectura Web pero con perfiles complementarios, uno de ellos con amplia experiencia en el desarrollo de sitios Web y experiencia en el análisis de accesibilidad y diseño de interfaces gráficas de usuario. El segundo experto, además de conocimientos técnicos, aportaba experiencia en la gestión universitaria, especialmente en el ámbito de gestión académica. Se contó igualmente con la colaboración y asesoramiento de un miembro del personal del servicio de informática de la propia universidad, implicado en proyectos de e-administración.

Para el análisis de las sedes electrónicas se diseñó un cuestionario de evaluación con 70 indicadores agrupados en 5 apartados o criterios básicos de evaluación definidos a partir de los requisitos básicos de lo que debería ser una sede electrónica universitaria eficiente (figura 1).

En concreto, los 5 criterios básicos con sus respectivos indicadores de evaluación son los que se muestran a continuación:

- Aspectos generales: con los 6 indicadores que se muestran en la tabla I, justificados por la necesidad de tener un acceso simple y reconocible a la sede y cumplir una serie de requerimientos formales establecidos por la legislación.

- Contenidos y servicios: con los 17 indicadores que se muestran en la tabla II. Para la elección de estos indicadores se tomaron como referencia el listado con contenidos y servicios mínimos de sedes electrónicas citadas en el Artículo 6 del Real Decreto 1671/2009 que lleva a la práctica la LAECSP, y las normas del marco regulador de una sede electrónica mencionadas en (Martínez, 2009). 


\section{FIGURA 1}

\section{Requisitos básicos de una sede electrónica universitaria}

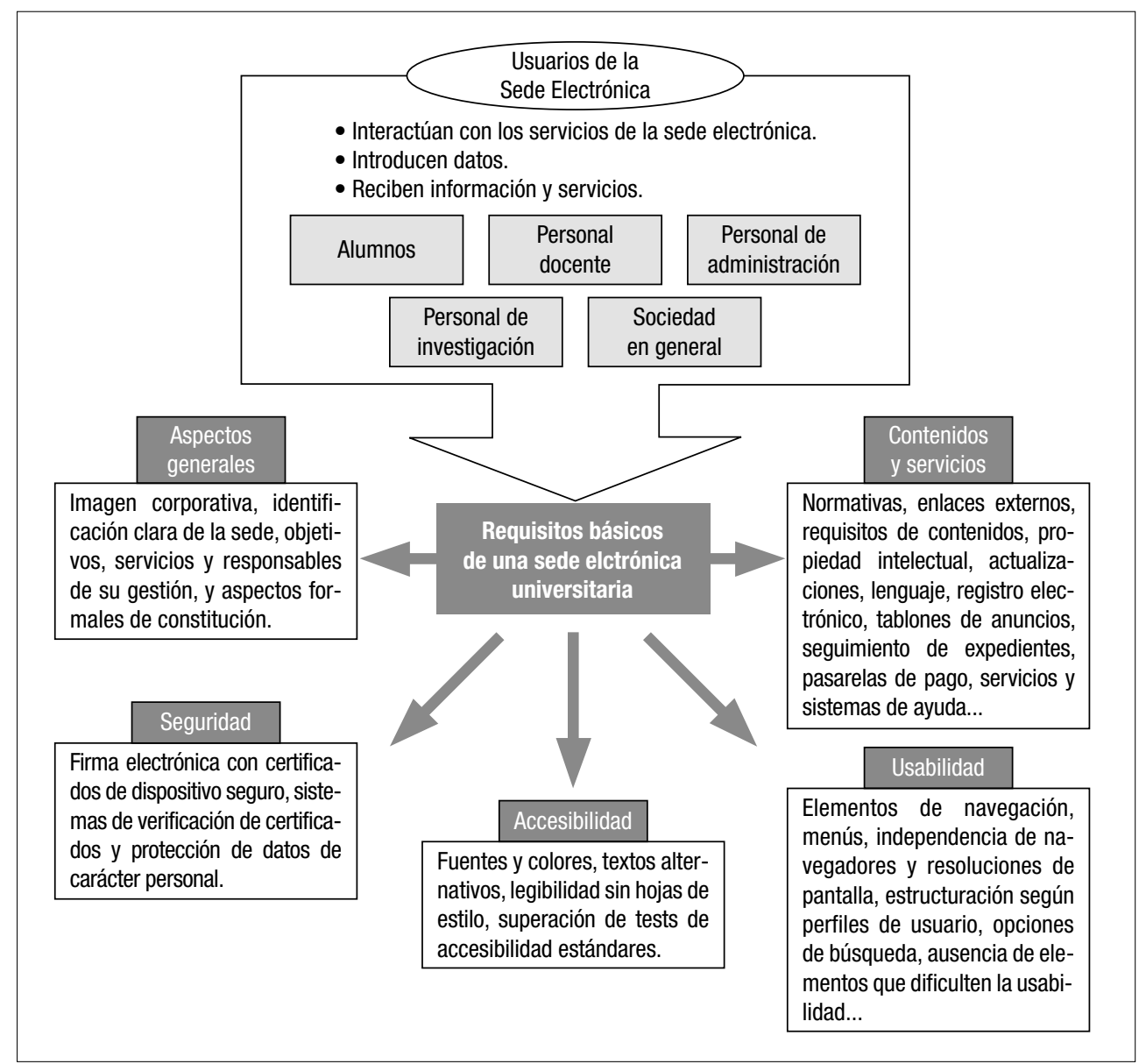

TABLA I

Indicadores de evaluación de los aspectos generales de las sedes electrónicas

(C1.1) Acceso claro desde el portal de la institución.

(C1.2) URL significativa e identificable.

(C1.3) Se identifican claramente el titular de la sede, sus objetivos y los responsables o encargados de su gestión.

(C1.4) Existe una sección específica de los servicios públicos electrónicos que oferta la sede.

(C1.5) Se ha publicado en la sede la orden o resolución por la que se crea.

(C1.6) Presencia de la imagen corporativa en todas las páginas. 


\section{TABLA II}

\section{Indicadores de evaluación de los contenidos y servicios de las sedes electrónicas}

(C2.1) Dispone de un repertorio de la normativa básica reguladora de la Administración.

(C2.2) Dispone de una sección que "conecte" mediante "Vínculos" con los Boletines Oficiales de referencia para la Administración titular de la sede.

(C2.3) Incluye el Perfil del Contratante.

(C2.4) Se muestra en la sede información relacionada con propiedad intelectual.

(C2.5) Se indica la relación de los medios electrónicos a los que se refiere el artículo 27.4 de la Ley 11/2007, de 22 de junio.

(C2.6) Incluye información sobre actualizaciones del portal.

(C2.7) Lenguaje entendible y cercano al usuario, con párrafos cortos de no más de 2 frases.

(C2.8) Posee un registro electrónico activo o una sección que dirija al mismo.

(C2.9) Incluye un tablón electrónico de anuncios o edictos.

(C2.10) Permite realizar consultas sobre el estado de tramitación de expedientes en los que tengan la condición de interesado.

(C2.11) Indicación de la fecha y hora oficial a los efectos previstos en el artículo 26.1 de la Ley 11/2007, de 22 de junio.

(C2.12) Se utilizan las lenguas co-oficiales.

(C2.13) Permite el pago de tasas a través de la sede.

(C2.14) Dispone de la relación de las direcciones de correo electrónico institucionales activas e información de contacto.

(C2.15) Existe una oficina virtual de ayuda.

(C2.16) Existe una sección para la formulación de quejas y sugerencias por parte de los administrados.

(C2.17) Colocación adecuada de la ayuda.

- Seguridad: con los 5 indicadores que se muestran en la tabla III. De nuevo, estos indicadores están justificados por las exigencias establecidas en el Artículo 6 del Real Decreto 1671/2009.

\section{TABLA III}

Indicadores de evaluación de los aspectos de seguridad de las sedes electrónicas

(C3.1) Dispone de sistemas de comunicación seguros (firma electrónica con certificados de dispositivo seguro o medio equivalente).

(C3.2) Se indican los sistemas de firma electrónica admitidos en la sede.

(C3.3) Tiene sistemas de verificación de certificados.

(C3.4) Muestra información relacionada con la protección de datos de carácter personal.

(C3.5) Incluye un enlace con la sede electrónica de la Agencia Española de Protección de Datos. 
- Accesibilidad: con los 12 indicadores que se muestran en la tabla IV. En este caso, se tomaron como referencia indicadores recogidos en varios trabajos de evaluación de accesibilidad de sitios Web: (García, 2010) (Ribera y otros, 2009) (Hoy-Yan y Zaphiris, 2003) y (Travieso y otros, 2007). Además, se trató de analizar la verificación del grado de cumplimiento de los tres niveles de prioridad en accesibilidad definidos por el World Wide Web Consortium (W3C) por medio del comité Web Accessibility Initiative (WAI). La última legislación española reglamenta que a partir de 31 de diciembre de 2008 todos los sitios Web dependientes de las administraciones públicas han de ser accesibles no solo a un nivel A, sino en el nivel superior AA (prioridad 2 de la Norma UNE 139803:2004). Por todo ello se consideró oportuno aplicar a las sedes electrónicas diferentes tests de accesibilidad Web, validación de códigos y análisis de visibilidad de las sedes electrónicas. En concreto se realizaron los tests:

- Test TAW para las prioridades I, II y III (http://www.tawdis.net/).

- HTML validator (http://validator.w3.org/).

- CSS validator (http://jigsaw.w3.org/css-validator/).

- Page Rank de Google de visibilidad Web (http://page-rank-calculator. com).

\section{TABLA IV}

Indicadores de evaluación de la accesibilidad de las sedes electrónicas

(C4.1) Posibilidad de ajuste del tamaño del texto.

(C4.2) Elección adecuada de colores: color de fondo con contraste con el texto.

(C4.3) Existencia de textos alternativos para elementos no textuales.

(C4.4) No dependencia de colores en la información requerida para navegar.

(C4.5) Existencia de una versión solo texto.

(C4.6) Se puede imprimir la página sin problemas y el resultado es legible.

(C4.7) Supera el test TAW de prioridad 1.

(C4.8) Supera el test TAW de prioridad 2.

(C4.9) Supera el test TAW de prioridad 3.

(C4.10) Supera el validador de HTML.

(C4.11) Supera el validador de CSS.

(C4.12) Visibilidad Web del portal.

- Usabilidad: con los 30 indicadores que se muestran en la tabla V. Para la elección de estos indicadores se tuvieron en cuenta las propuestas metodológicas de evaluación de usabilidad y accesibilidad recogidas en (Nielsen, 2003) (García, 2004) y (Travieso y otros, 2007). En los aspectos de usabi- 


\section{TABLA V}

\section{Indicadores de evaluación de la usabilidad de las sedes electrónicas}

(C5.1) Tiene mapas de navegación interactiva para su correcta utilización y navegación.

(C5.2) Posee un sistema de navegación intuitivo.

(C5.3) Incluye vínculos de navegación en el pie de página.

(C5.4) Ausencia de redireccionamiento en la entrada al portal.

(C5.5) Identificación clara de los elementos enlazados.

(C5.6) Posibilidad de retorno directo a la página de inicio en todo momento.

(C5.7) Menú permanente con no más de 7 opciones.

(C5.8) Disponibilidad de una barra de "rastros" en todo momento.

(C5.9) Links externos se abren en nuevas ventanas.

(C5.10) Presenta menús con títulos y textos concisos y explicativos.

(C5.11) Los iconos utilizados son comprensibles.

(C5.12) Dimensiones óptimas de la página.

(C5.13) Se observa correctamente con diferentes resoluciones de pantalla.

(C5.14) Se aprovecha equilibradamente el espacio visual de la página para evitar la sobresaturación de elementos, con espacios en blanco (libres) entre el contenido, para descansar la vista.

(C5.15) Se ofrece información sobre limitaciones o condiciones para la navegabilidad (límite de usuarios, necesidad de registrarse, fechas de caducidad para el acceso, costos de las operaciones).

(C5.16) Se especifican los aspectos técnicos para una correcta presentación (plug-ins, versión del navegador, resolución de pantalla).

(C5.17) Estructura organizada con criterio de perfil de usuario.

(C5.18) Tiempo de descarga adecuado (máximo de 10 segundos).

(C5.19) Ausencia de marcos.

(C5.20) El rotulado es significativo, correcto y utiliza lemas estándares: "Mapa del sitio", "Acerca de", "Quiénes somos", "Ayuda", "FAQs", "Regístrese"

(C5.21) Existencia de una opción de búsqueda en la parte superior de la página principal.

(C5.22) Existencia de una opción de búsqueda avanzada.

(C5.23) No necesidad de plug-ins para visualizar las páginas.

(C5.24) Ausencia de elementos multimedia como animaciones o música.

(C5.25) Imágenes/Ilustraciones ocupando entre el 5 y el 15\% del espacio de la página de inicio.

(C5.26) Fuente de texto adecuada (negro, 12pt, sans-serif).

(C5.27) Títulos descriptivos de las páginas (etiqueta TITLE).

(C5.28) Ausencia de ventanas emergentes (pop-ups).

(C5.29) Ausencia de menús desplegables, texto móvil, marquesinas, texto flotante...

(C5.30) Independencia del navegador y su versión.

554 Rev. Esp. Doc. Cient., 34, 4, octubre-diciembre, 545-562, 2011. ISSN: 0210-0614. doi: 10.3989/redc.2011.4.845 
lidad, buen número de los criterios seleccionados están igualmente recogidos en la guía de evaluación heurística desarrollada por Hassan y Martín para facilitar la evaluación de la usabilidad de sitios Web (Hassan y Martín, 2003).

Si bien la mayoría de los criterios son de carácter cualitativo, como ayuda para la evaluación se aportaron a los evaluadores pautas para otorgar valoraciones cuantitativas (entre 0 y 3 ) de cada uno de los indicadores analizados. Algunos de los indicadores están establecidos a modo de "checklist" en los que la respuesta puede ser afirmativa o negativa, como criterio general, en el primer caso se otorga la puntuación 3 y en el segundo la puntuación 0. En otros, en cambio, se consideró oportuno permitir al evaluador otorgar valoraciones cuantitativas en el rango $[0,3]$ para valorar el grado de cumplimiento de determinado criterio en cada una de las sedes electrónicas. Por ejemplo, el indicador C2.1 hace referencia a la existencia de un repertorio de la normativa básica reguladora de la administración, no se trata simplemente de valorar la existencia o no de ese repertorio sino en cierta forma medir lo completo que es.

A la vista de los 70 indicadores, se puede comprobar la necesidad de contar con evaluadores con experiencia no solo en aspectos técnicos relacionados con diseño Web sino también con un buen conocimiento del funcionamiento de los procesos de gestión universitaria.

Cada uno de los evaluadores examinó de manera independiente cada una de las 17 sedes electrónicas, para posteriormente poner en común los problemas detectados y elaborar un informe final consensuado de acuerdo a los criterios de Hassan y Ortega (Hassan y Ortega, 2009). El informe de evaluación de cada sede incluía una valoración cuantitativa de cada uno de los 70 indicadores y el valor promedio para cada uno de los cinco criterios principales que a su vez fueron agregados en un valor promedio final. Debe aclararse que el propósito de este trabajo no es tanto medir el número y calidad de los trámites administrativos que pueden realizarse a través de cada una de las sedes, sino más bien verificar el correcto diseño y estructura de la sede bajo parámetros de usabilidad, accesibilidad y requerimientos formales fijados por las regulaciones específicas.

\section{Análisis de resultados}

Tras la valoración cuantitativa de los 70 indicadores por parte de los expertos y su agregación en una valoración de cada uno de los 5 criterios principales, los resultados obtenidos son los que se muestran en la tabla VI. Dicha tabla identifica las 17 universidades cuyas sedes electrónicas han sido analizadas y las ordena de acuerdo a la puntuación global obtenida en el análisis. Debe indicarse que la más alta valoración de una sede no implica que ofrezca un mayor número de servicios, sino más bien que cumple en mayor medida los aspectos formales de lo que se considera una sede electrónica eficiente. 


\section{TABLA VI}

Valoración de las sedes electrónicas universitarias españolas (febrero 2011)

\begin{tabular}{|c|c|c|c|c|c|c|}
\hline Universidad & $\begin{array}{c}\text { Puntuación } \\
\text { global }\end{array}$ & $\begin{array}{l}\text { Aspectos } \\
\text { generales }\end{array}$ & $\begin{array}{l}\text { Contenidos } \\
\text { y servicios }\end{array}$ & Accesibilidad & Seguridad & Usabilidad \\
\hline $\begin{array}{l}\text { Politécnica de Car- } \\
\text { tagena }\end{array}$ & 1,883 & 2,667 & 1,647 & 1,333 & 1,500 & 2,267 \\
\hline Jaén & 1,861 & 2,000 & 1,412 & 1,778 & 1,750 & 2,367 \\
\hline Granada & 1,854 & 1,500 & 1,471 & 2,333 & 1,500 & 2,467 \\
\hline Córdoba & 1,816 & 2,167 & 0,882 & 2,000 & 1,500 & 2,533 \\
\hline Murcia & 1,812 & 2,500 & 1,706 & 1,222 & 1,000 & 2,633 \\
\hline Huelva & 1,763 & 2,000 & 1,000 & 1,667 & 1,750 & 2,400 \\
\hline Pablo de Olavide & 1,733 & 1,667 & 1,706 & 1,444 & 1,750 & 2,100 \\
\hline $\begin{array}{l}\text { Politécnica de Ca- } \\
\text { talunya }\end{array}$ & 1,730 & 2,333 & 0,941 & 1,444 & 1,500 & 2,433 \\
\hline Sevilla & 1,658 & 1,667 & 1,235 & 1,889 & 1,500 & 2,000 \\
\hline Málaga & 1,648 & 1,667 & 1,353 & 1,556 & 1,500 & 2,167 \\
\hline $\begin{array}{l}\text { Politécnica de Va- } \\
\text { lencia }\end{array}$ & 1,600 & 2,167 & 1,294 & 0,889 & 1,250 & 2,400 \\
\hline Almería & 1,581 & 2,000 & 1,118 & 1,556 & 1,000 & 2,233 \\
\hline Valencia & 1,397 & 1,333 & 1,118 & 1,333 & 1,500 & 1,700 \\
\hline Rovira i Virgili & 1,344 & 2,000 & 0,588 & 1,667 & 0,000 & 2,467 \\
\hline León & 1,241 & 2,000 & 0,706 & 1,333 & 0,000 & 2,167 \\
\hline Pompeu Fabra & 1,240 & 1,833 & 0,529 & 1,556 & 0,250 & 2,033 \\
\hline $\begin{array}{l}\text { Politécnica de Ma- } \\
\text { drid }\end{array}$ & 1,153 & 1,333 & 0,412 & 1,889 & 0,000 & 2,133 \\
\hline
\end{tabular}

De las 5 sedes mejor valoradas, la de la Universidad Politécnica de Cartagena (figura 2) obtiene una valoración promedio más alta en los 5 criterios, la de la Universidad de Granada destaca en aspectos de accesibilidad, la de Jaén en los aspectos relacionados con seguridad y protección de datos, y la Universidad de Murcia es la que mejor valoración obtiene en aspectos de usabilidad.

La figura 3 muestra la valoración de las 17 sedes analizadas, ordenadas de acuerdo a su valoración global. En la figura 4 pueden verse, además, las puntuaciones medias de las 17 sedes en cada uno de los 5 criterios básicos de evaluación. Como puede apreciarse, la valoración en aspectos de usabilidad es la más destacada, en cambio, en promedio, se obtienen peores evaluaciones en los temas relacionados con la seguridad y la información y servicios proporcionados al usuario. Algunas de las sedes electrónicas evaluadas, se encontraban en la fecha del análisis en proceso de consolidación, lo que puede justificar esa valoración media. 
FIGURA 2

Portal Web de la Universidad Politécnica de Cartagena y su sede electrónica

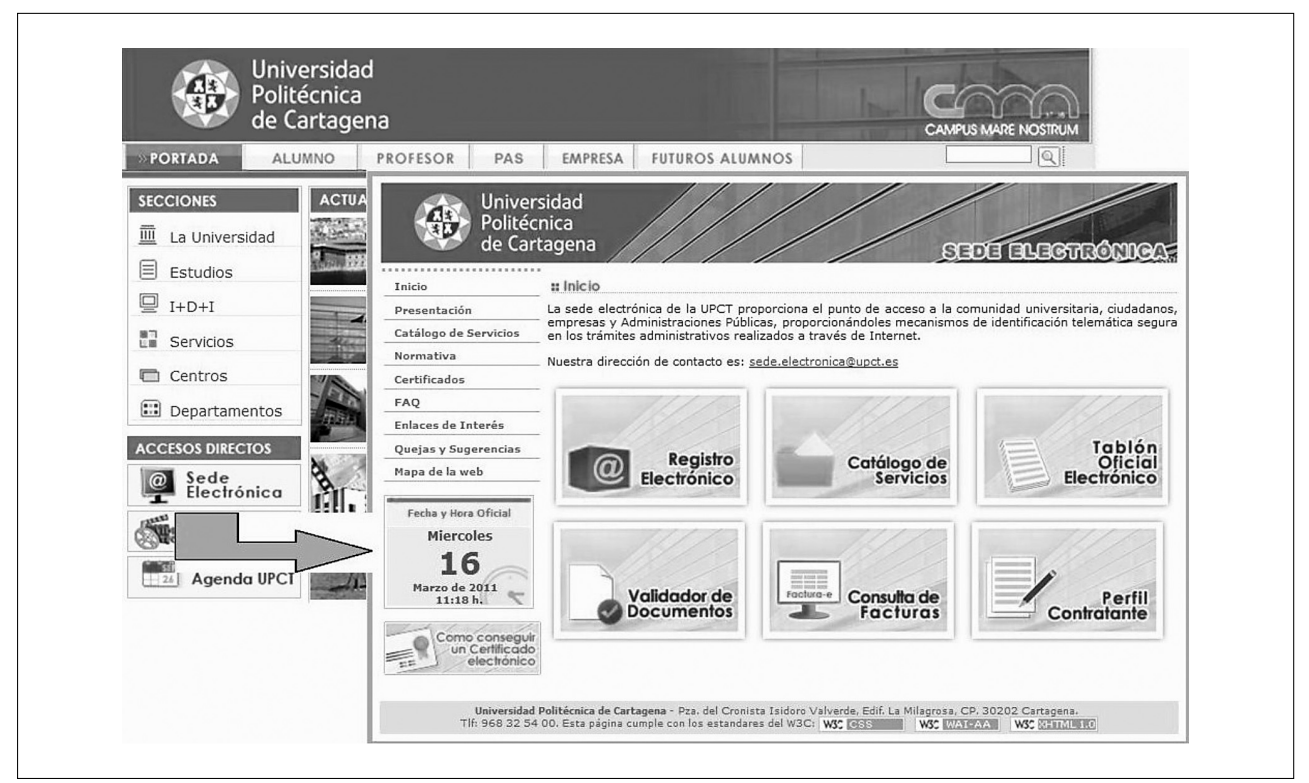

FIGURA 3

Valoración global de los criterios de evaluación de las sedes electrónicas universitarias

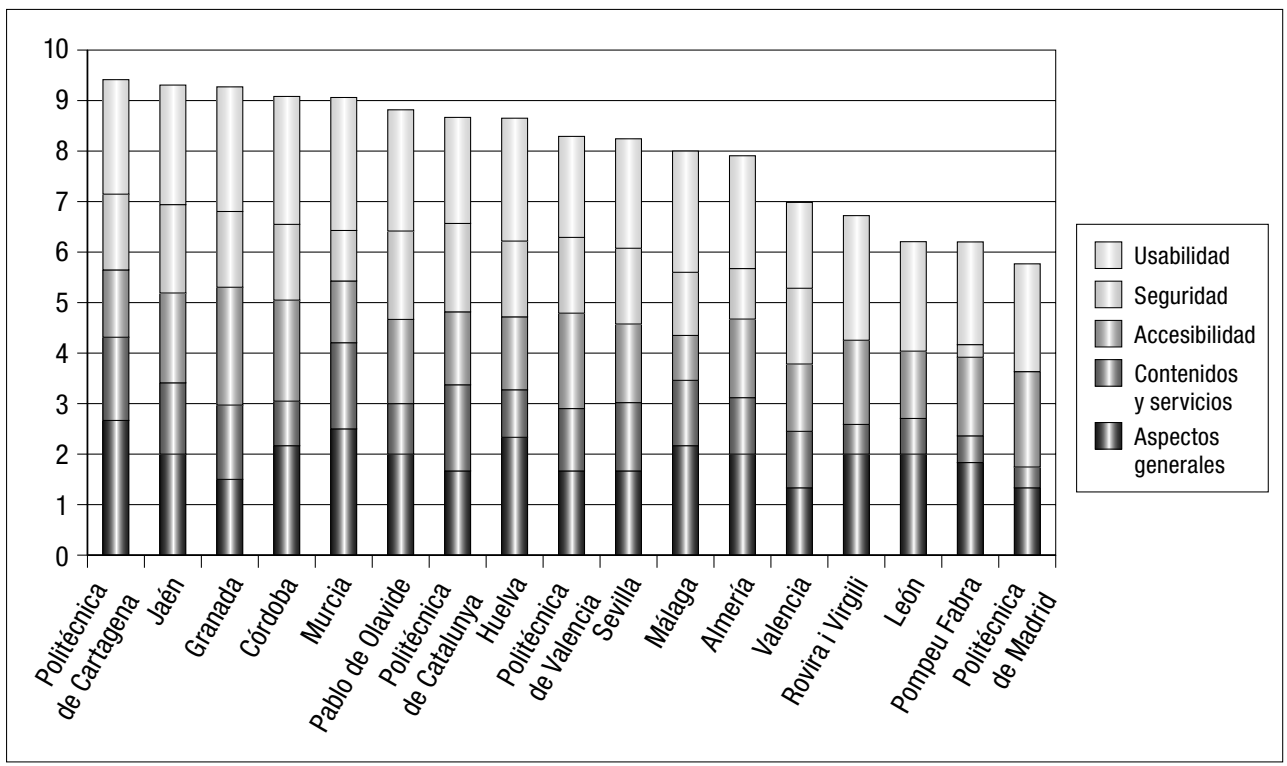

Rev. Esp. Doc. Cient., 34, 4, octubre-diciembre, 545-562, 2011. ISSN: 0210-0614. doi: 10.3989/redc.2011.4.845 
FIGURA 4

Valoraciones promedio de los 5 criterios básicos de evaluación

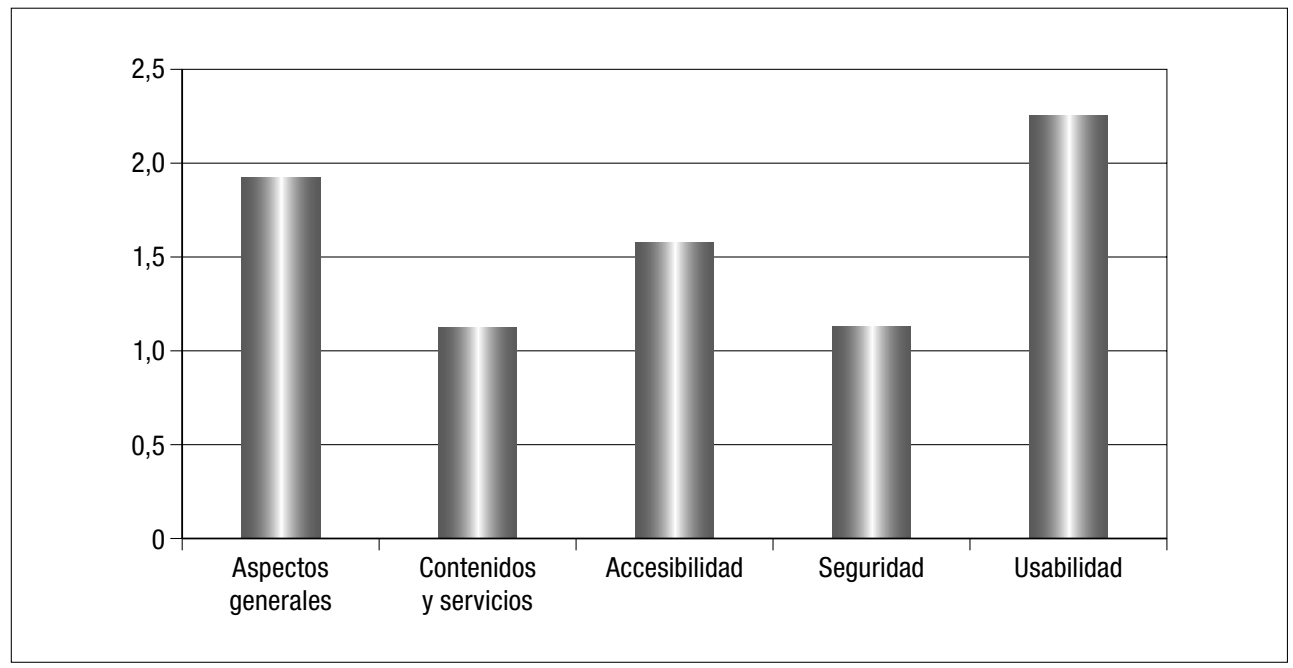

De los resultados de la valoración de los 70 indicadores por los expertos se pueden destacar también algunos datos interesantes:

- Criterio de aspectos generales: En general, el acceso a las sedes electrónicas es claro y sencillo desde el portal Web de la propia universidad, la mayoría de las universidades con sede electrónica (76\%) tienen un enlace visible en un lugar destacado de la página principal con referencia expresa al término "Sede electrónica" o "Administración electrónica". Solo en 4 de las sedes analizadas el acceso resulta un tanto confuso. En el $71 \%$ de los casos es posible acceder directamente a través de un URL identificable y sencillo, que es fácilmente vinculable con la propia institución. Un aspecto a destacar negativamente es que son escasas las sedes que incluyen información sobre la orden o resolución por la que se crean, únicamente 3 de las sedes analizadas lo hacen.

- Criterio de contenidos y servicios: El Artículo 6 del RD 1.671/2009 marca unos contenidos y servicios mínimos que deberían tener las sedes electrónicas. No obstante, el grado de cumplimiento de esos requisitos es variable en las sedes analizadas, algunas de ellas están aún en proceso de construcción y en otras no están presentes algunos elementos que la regulación exige. Aunque la mayoría de las sedes incluyen una sección con la normativa básica reguladora, solo un 6\% incluye enlaces a boletines oficiales de referencia para la administración. Una única sede incluye información sobre propiedad intelectual. Son también escasas (24\%) las sedes que muestran información sobre sus últimas actualizaciones. La fecha y hora oficial tam- 
poco se muestra en todas las sedes, el 65\% de las sedes son las que cumplen este requisito fijado por el RD 1.671/2009.

El perfil del contratante está presente en poco más de la mitad de las sedes (53\%), también aproximadamente la mitad cuentan con un registro electrónico activo. Solo el $24 \%$ cuentan con un tablón electrónico para la publicación de anuncios. También resultan una minoría (4 sedes, 24\%) las que disponen de una pasarela de pago integrada en la sede para realizar pago de tasas directamente dentro de la sede.

Respecto a la ayuda en el manejo de la sede, solo un 29\% de las sedes tienen definida una oficina virtual de ayuda y el $41 \%$ cuentan con una sección específica para la formulación de quejas y sugerencias.

- Criterio de seguridad: Los aspectos técnicos de seguridad están en general bien cubiertos en las sedes electrónicas universitarias, con sistemas de comunicación seguros, sistemas de verificación de certificados y firma electrónica. Pero fallan principalmente en la información que trasladan a los usuarios sobre esos sistemas de seguridad. Otro aspecto que destaca negativamente es la escasa información que las sedes proporcionan sobre protección de datos de carácter personal, solo un 24\% incluye alguna información. Además, ninguna incluye un vínculo con la Agencia Española de Protección de Datos, tal como exige el Artículo 6 del RD $1.671 / 2009$.

- Criterio de accesibilidad: Para estudiar el nivel de accesibilidad de las sedes electrónicas de las universidades públicas españolas se pasó el Test TAW a cada una de las sedes. Dicho test mide la cantidad de pautas que supera de forma automática y hace posible conocer el nivel de accesibilidad de los sitios Web, sin que esto signifique necesariamente que el sitio analizado pueda considerarse accesible. No obstante, estos indicadores pueden ser útiles para obtener una visión general sobre el grado de preocupación por temas de accesibilidad en las sedes electrónicas. El test TAW analizó los tres niveles de prioridad: nivel 1 de cumplimiento requerido, nivel 2 de cumplimiento recomendado y nivel 3 de cumplimiento optativo. El 65\% de las sedes no reportaron ningún error de accesibilidad de detección automática de nivel 1, y las restantes sedes tuvieron un número de errores de nivel 1 mínimo, por lo que se puede decir que el nivel 1 está prácticamente superado en todas las sedes. En lo que se refiere al nivel 2, que también debería ser de obligado cumplimiento, son 7 las sedes que pasan el test, lo que supone un 41\%; 6 de ellas superan igualmente el test de nivel 3. Un problema de accesibilidad recurrente en todas las sedes analizadas es la ausencia de una versión solo texto. Parece, por tanto, a la vista de estos resultados que queda pendiente de realizar un trabajo de adaptación para alcanzar las previsiones oficiales de requerimientos de accesibilidad en la administración pública. Conclusiones que coinciden con las obtenidas por Ribera, Térmens y Frías en su estudio sobre accesibilidad de las webs de las universidades españolas (Ribera y otros, 2009). 
Respecto a los otros tests de validación realizados a las sedes electrónicas, se podría decir que el $41 \%$ de ellas superan exitosamente el validador de HTML sin ningún error. En el caso de validador de hojas de estilo son solo 3 sedes las que no plantean ningún error, lo que supone un 18\%.

Finalmente, se analizó también la visibilidad de las sedes a través del nivel de Page Rank de Google, resulta destacado el hecho que si bien la visibilidad de los portales Web de las universidades es alta, un 7,8 de media, a la hora de cuantificar la visibilidad de sus sedes electrónicas el valor medio baja hasta 2,7. El Page Rank se calcula sobre la base de la visibilidad de las páginas Web medida a través de los enlaces que reciben y la importancia de las páginas de origen de dichos enlaces, en el caso de las sedes electrónicas muchas de ellas aún no están teniendo el uso que potencialmente podrían tener.

- Criterio de usabilidad: En aspectos de usabilidad se podría destacar que todas las sedes cuentan con sistemas de navegación intuitivos, no obstante, los mapas de navegación Web están presentes solo en el 47\% de las sedes, y las barras de rastros en el $65 \%$. Un $47 \%$ de las sedes muestran información sobre requerimientos técnicos. Son pocas las sedes que cuentan con opciones de búsqueda avanzada (29\%). Prácticamente todas las sedes se visualizan correctamente con independencia del navegador y los tiempos de descarga son adecuados. No obstante, en algunas de ellas se dificulta la visualización y navegación cuando se trabaja a resoluciones de pantalla bajas o requieren excesivos desplazamientos del área visible. En general, se podría decir que las sedes electrónicas universitarias cuentan con unos buenos niveles de usabilidad.

\section{Conclusiones}

A la vista de los análisis realizados se puede concluir que está clara la apuesta del sistema universitario español por la e-administración, con una clara progresión de crecimiento en el nivel de implantación, aunque hay aún un notable camino por recorrer. La actual coyuntura económica y los profundos cambios que la integración en el EEES está exigiendo a las universidades han venido a dificultar la evolución de la administración universitaria hacia una verdadera administración electrónica. No obstante, dentro de las administraciones públicas, las universidades están demostrando ser instituciones pioneras en la puesta en marcha de estos procesos, con un compromiso claro por cumplir los requisitos de una verdadera administración del siglo XXI.

En la actualidad se observa una desigual penetración de la administración electrónica en las universidades, con algunas Comunidades Autónomas que han realizado una apuesta clara por esta nueva forma de relacionarse con los usuarios y han apoyado a sus universidades. Este es el caso de las universidades catalanas y andaluzas, principalmente. Llamativa también es la situación de las universida- 
des murcianas, con una gran preocupación por la modernización de sus procesos de administración electrónica.

En la evaluación de las sedes electrónicas universitarias actualmente existentes se ha podido observar que, en general, se está en un proceso que requiere una mayor consolidación. Algunas sedes ofrecen aún una cantidad limitada de servicios y se observan con carácter general algunas carencias o incumplimientos de aspectos formales establecidos por la legislación española sobre administración electrónica. En general, los aspectos técnicos analizados se encuentran bastante bien cubiertos, pero donde queda camino por recorrer es en la generalización de los servicios ofertados a todo tipo de usuarios y no solo a personal y estudiantes de las propias universidades. Aunque la CRUE trata de promover la implantación de la e-administración en las universidades españolas, se hace necesaria una mayor coordinación y cooperación entre ellas. Especialmente si se tienen en cuenta aspectos como la movilidad promovida por el EEES, los nuevos conceptos de reconocimiento o transferencia de créditos, la cada vez más frecuente aparición de programas formativos interuniversitarios, los procesos cada vez más complejos y competitivos de acceso a la universidad, etc. En todos ellos la e-administración tiene un enorme potencial que aún no están aprovechando las universidades.

Si bien se puede observar que solo una tercera parte de las universidades públicas han implantado sedes electrónicas, prácticamente todas tienen grupos de trabajo constituidos y es de esperar que en los próximos años se vea una implantación generalizada de sedes electrónicas universitarias. Éstas facilitarán el acceso a la información a los usuarios y agilizarán los procesos vinculados a los servicios que brindan las universidades. En paralelo, los diferentes usuarios irán familiarizándose con el uso de estos servicios y descubriendo las ventajas que la e-administración proporciona.

\section{Bibliografia}

Aibar, E.; Urgell, F., y Welp, Y. (2004). ¿De los portales públicos a la administración red? Un estudio de caso sobre e-governance. e-Administración. II congrés online de l'Observatori per la CiberSocietat, España.

Bárcena, I. (2010). Una plataforma común para la e-Administración universitaria de Cataluña. Boletín de RedIRIS, número 88-89. Disponible en: http://www.rediris.es/difusion/ publicaciones/boletin/88-89/Ponencia5.C.pdf [consulta: 11-02-2011].

García, J. C. (2004). Usabilidad de las páginas de inicio de los diarios digitales españoles. Scire, vol. 10 (2), 9-31.

García, J. C. (2010). Barreras para el acceso al contenido de las webs de universidades españolas. Scire, vol. 16 (1), 47-63.

González, M. P.; Pascual, A., y Lorés, J. (2001). Evaluación Heurística. En: Lorés, J. (ed.), Introducción a la Interacción Persona-Ordenador. AIPO: Asociación Interacción Persona-Ordenador. Disponible en: http://www.aipo.es/libro/libroe.php [consulta: 20-4-2011]. 
Guillén, J. (2010). La Administración Electrónica: ¿Mito o realidad para los ciudadanos del siglo XXI? Centro PwC \& IE del Sector Público, ISBN 978-84-693-4507-8. Madrid.

Hassan, Y., y Martín, F. J. (2003). Guía de Evaluación Heurística de Sitios Web. No Solo Usabilidad, $\mathrm{n}^{\circ}$ 2. Disponible en http://www.nosolousabilidad.com [consulta: 20-4-2011].

Hassan, Y., y Ortega, S. (2009). Informe APEI sobre usabilidad (Informe APEI 3). Disponible en: http://www.nosolousabilidad.com [consulta: 20-4-2011].

Hoi-Yan, T. M., y Zaphiris, P. (2003). The usability and content accessibility of the e-government in the UK. En: C. Stephanidis (ed.), Universal access in HCI, Lawrence Erlbaum, Mehwah, USA, pp. 760-764.

Martínez, R. (2009). Una nueva sede del poder: Las Sedes Electrónicas. MULTITEC: Administración Electrónica: la Ley y la realidad. Disponible en: http://labje.unizar.es/ UIMP3/Ponencias/sede_del_poder.pdf [consulta: 11-02-2011].

Nielsen, J. (2003). Usability 101: Introduction to Usability. Disponible en: http://useit.com/ alertbox/20030825.html [consulta: 11-02-2011].

Ribera, M.; Térmens, M., y Frías, A. (2009). La accesibilidad de las webs de las universidades españolas. Balance 2001-2006. Revista Española de Documentación Científica, vol. 32 (3), 66-88.

Travieso, C.; Alonso, J., y Vivancos, J.M. (2007). Usabilidad de los catálogos de las bibliotecas universitarias: propuesta metodológica de evaluación. Acimed, vol. 16 (2).

UniversitiC 2010. (2010). Evolución de las TIC en el sistema universitario español 20062010. Conferencia de Rectores de las Universidades Españolas (CRUE). Disponible en: http://www.crue.org/Publicaciones/universitic.html [consulta: 11-02-2011]. 